\title{
The HPV-activating Cellular Transcription Factor Brn-3a is Overexpressed in CIN3 Cervical Lesions
}

\author{
Daniel Ndisdang, ${ }^{*}$ Peter J. Morris, ${ }^{*}$ Caroline Chapman, ${ }^{*}$ Linda Ho, ${ }^{*}$ Albert Singer, ${ }^{\ddagger}$ and David S. Latchman \\ *Windeyer Institute of Medical Sciences, University College London Medical School, The Windeyer Building, Cleveland Street, London \\ W1P 6DB, United Kingdom; and Department of Women’s and Children’s Health, The Whittington Hospital, Highgate Hill, London N19 \\ $5 N F$, United Kingdom
}

\begin{abstract}
The cervical cellular transcription factors Brn-3a and Brn-3b have antagonistic effects on transcription of the human papilloma virus types 16 and 18 E6 and E7 oncogenes, with Brn-3a activating expression and Brn-3b repressing it. We therefore measured expression of Brn-3a and Brn-3b mRNAs in biopsies from 16 women with no detectable cervical abnormality, and in 14 women with cervical intraepithelial neoplasia grade 3 (CIN3) lesions. Although the mean level of Brn-3b expression was similar in both groups, the mean level of Brn-3a expression was over 300-fold higher in the CIN3 samples when compared with normals. Elevated expression of Brn-3a was also detected in 16 histologically normal regions of the cervix adjacent to the CIN3 lesions, indicating that elevation of Brn-3a levels is not confined to the lesion in women with CIN3, and is thus not a consequence of the oncogenic process. The elevated levels of Brn-3a in the CIN3 patient samples, together with the activating effect of Brn-3a on HPV-16 and -18 oncogene expression, suggest that induction of this factor is involved in activating HPV-16 and -18 oncogene expression in the cervix, and hence in the production of cervical cancers induced by HPV. ( $J$. Clin. Invest. 1998. 101:1687-1692.) Key words: cervical cancer - human papillomavirus - transcriptional regulation • Brn-3a and Brn-3b transcription factors
\end{abstract}

\section{Introduction}

The human papillomavirus types 16 and 18 (HPV-16 and HPV -18$)^{1}$ are found in the great majority of women with cervical cancer $(1,2)$, and it is now generally accepted that these oncogenic viruses play a critical role in the etiology of the disease. However, both HPV-16 and HPV-18 can be detected in

Address correspondence to David S. Latchman, Windeyer Institute of Medical Sciences, University College London Medical School, The Windeyer Building, Cleveland Street, London W1P 6DB, United Kingdom. Phone: 44-171-380-9343; FAX: 44-171-387-3310; E-mail: d.latchman@ucl.ac.uk

Received for publication 1 July 1997 and accepted in revised form 21 January 1998.

1. Abbreviations used in this paper: $\mathrm{CIN} 3$, cervical intraepithelial neoplasia grade 3; HPV, human papillomavirus; N-CIN, normal samples from regions adjacent to CIN3 lesions; RT, reverse transcription; URR, upstream regulatory region.

J. Clin. Invest.

(C) The American Society for Clinical Investigation, Inc. 0021-9738/98/04/1687/06 \$2.00

Volume 101, Number 8, April 1998, 1687-1692

http://www.jci.org women with undetectable or minimal cervical abnormality (3, 4), and it is unclear what factors trigger HPV-16 or HPV-18 to initiate cellular transformation to a malignant phenotype.

The ability of HPV-16 and HPV-18 to initiate cell transformation is dependent on the virally encoded E6 and E7 oncoproteins, each of which have a role in cell transformation in culture. In turn, transcription of the genes encoding E6 and E7 is controlled by an upstream regulatory region (URR) of the virus genome that is preferentially active in cells of cervical origin $(5,6)$. Although numerous studies have documented binding of several cellular transcription factors to the URR (7-10), these factors are generally ubiquitously expressed and may not fully explain cervical cell-specific activity of the URR or its activation leading to disease progression in vivo.

We previously reported, however, that a cervical cell-specific regulatory activity was able to bind to a sequence (ATGCAATT) in the URR of HPV-16 and HPV-18, and to activate their expression specifically in cell lines of cervical origin (11, 12). This sequence was absent in the URR of closely related HPVs such as HPV-6 and HPV-11, which cause only benign warts. We subsequently showed that this activity is mediated by two distinct transcription factors (Brn-3a and Brn-3b) that are expressed in cervical cells and neuronal cells, but not in other cell types (12). Brn-3a (also known as Brn-3.0:-; 13, 14) and Brn-3b (also known as Brn-3.2:-; 14, 15) are members of the POU family of transcription factors (for review see references 16 and 17) that are closely related to one another, but are encoded by different genes (18). Most interestingly, while Brn-3a activates transcription via the ATGCAATT motif of the HPV URR, Brn-3b represses such transcription and interferes with activation by Brn-3a (19).

Brn-3a and Brn-3b thus constitute a pair of closely related transcription factors that are specifically expressed in cervical cells, and which have antagonistic effects on the activity of the URR. It is possible, therefore, that the balance between Brn-3a and Brn-3b in vivo may play a role in regulating the activity of the URR, and hence in controlling production of the oncogenic E6 and E7 proteins and in transforming cells to a malignant phenotype. We have therefore compared the expression of Brn-3a and Brn-3b in cervical material from women with no histologicaly detectable cervical abnormality, and in those with cervical intraepithelial neoplasia grade 3 (CIN3) lesions.

\section{Methods}

Cervical biopsies, plasmids, and HPV-16 DNA. Human cervical biopsies were obtained from women attending the Colposcopy Clinic (Whittington Hospital, Highgate Hill, United Kingdom) who were referred with abnormal cervical smears ranging from mild to severe dyskariosis. All women provided informed consent at the time of colposcopy. Ethical permission was obtained from the Whittington Hospital Ethical Committee after review of the study protocol. Samples to be tested were chosen to represent a range of histologic grades independent of HPV status. Biopsy was either by punch or loop exci- 
sion. Each specimen was divided into 1-mm segments. Alternate sections of the biopsies were either transported in liquid nitrogen and stored at $-70^{\circ} \mathrm{C}$ before nucleic acid extraction, or were sent for histology. Sections were histologically classified according to the presence of cervical intraepithelial neoplasia. High-grade lesions were those with a diagnosis of CIN2/3, and low-grade lesions were those with a diagnosis of CIN1. Normal tissues were also classified.

The biopsies were made up of (a) 14 CIN3 lesions (CIN3); (b) 16 normal samples from regions adjacent to CIN3 lesions (N-CIN); and (c) 16 normal samples from individuals with no histologically detectable cervical abnormality. Complementary DNA (cDNA) obtained from human neuroblastoma cell lines expressing Brn-3a and Brn-3b was used as a positive control for reverse transcription (RT)-PCR experiments. Water only or human fibroblast mRNA (which does not express Brn-3a or Brn-3b) was used as a negative control. Plasmids with human Brn-3a and Brn-3b cDNA were used as positive controls for PCR experiments. HPV-16 DNA was used as a positive control for subsequent HPV PCR procedures, with water, DNA, or RNA from an HPV-negative neuroblastoma cell line being used as a negative control.

$R N A$ and DNA extractions from human cervical biopsies and RT$P C R$. RNA was prepared using the guanidinium isothiocyanate method (20). Samples of $\sim 33 \mathrm{mg}$ were homogenized in $250 \mu \mathrm{l}$ denaturation solution containing $4 \mathrm{M}$ guanidinium thiocyanate, $25 \mathrm{mM}$ sodium citrate, $\mathrm{pH} 7,0.1 \mathrm{M}$ 2-mercaptoethanol, and $0.5 \% \mathrm{~N}$-laurolsarcosine. DNA was extracted using guanidinium isothiocyanate as previously described (21). Resultant RNA and DNA were treated with $250 \mathrm{mg} / \mathrm{ml}$ DNase and RNase, respectively. About $0.1 \mu \mathrm{g}$ of the resultant RNA from each sample was used as a template for cDNA synthesis. The synthesized cDNA was used in RT-PCR assays as previously described $(22,23)$ using the following oligonucleotide primers: Brn-3a: 5'GTCGACATGGACTCGGACACG-3', 3'-ACGGTGAATGACTCCCCCGA-5'; Brn-3b: 5'-GGAGAAGAAGCGCAAGC-3', 3'CTGAGAACCGGAGAGGTCT-5'. Amplification of the invariantly expressed human cyclophilin mRNA used as a control was carried out in parallel using the following primers: 5'-TTGGGCCGCGGTACTCCTTTCA-3', and 3'-TTTCGTATGGCCCAGGACCG-5' (22). HPV-16 DNA and cDNA prepared from HPV-16 mRNA was amplified using a hot-start of $95^{\circ} \mathrm{C}$ and an annealing temperature of $54^{\circ} \mathrm{C}$ for 35 cycles with the following primers: $5^{\prime}$-gagaagcttCTGCAATGTTTCAGGACCC-3', 3'-gactcgaGTGCCCATTAACAGGTCTTC-5' (21).

In all cases, $20 \mu \mathrm{l}$ of each PCR product was fractionated on a $2 \%$ agarose gel and blotted onto Hybond-N+ nylon membrane (Amersham International, Little Chalfont, United Kingdom) and hybridized with homologous complimentary ${ }^{32} \mathrm{P}$-labeled probes. Membranes were exposed to films, (Eastman Kodak Co., Rochester, NY) and the subsequent autoradiographs were then analyzed using a densitometer (Bio-Rad Laboratories, Hercules, CA). We have previously shown that this blotting procedure, in conjunction with the RTPCR conditions used, allows accurate quantification of the Brn-3a and Brn-3b mRNAs relative to the constitutively expressed cyclophilin mRNA $(14,22-25)$.

Western blotting. Samples for Western blot protein assay were made up of 11 CIN3 lesions, 10 normal samples from adjacent regions, and 13 normal samples from women with no histologically detectable abnormality. The samples were resuspended in sample buffer containing $2.3 \%$ sodium dodecyl sulphate, $0.0625 \mathrm{M}$ Tris $/ \mathrm{HCl}$, $\mathrm{pH} 7.9,10 \%$ glycerol, $5 \% \beta$-mercaptoethanol, and bromphenol blue. Samples were sonicated for $10 \mathrm{~s}$, and then boiled for $2 \mathrm{~min}$. The samples had equal protein content as determined by the method of Bradford (26), and were split in two and run on two sides of the same SDSpolyacrylamide gel. After electrophoresis, one half of the gel was stained with Coomassie blue and then destained, while the other half was transferred to nitrocellulouse and probed with antibody to Brn-3a or Brn-3b (Bab Co Ltd) as previously described (27). Levels of Brn-3a or Brn-3b in each sample were quantified by densitometric scanning of the resulting autoradiograph. Differences in the level of total pro- tein in each sample were determined by scanning the actin band on the stained portion of the gel to normalize the samples (27).

\section{Results}

To measure the Brn-3a and Brn-3b mRNAs in the limited amounts of material available from human cervical biopsies, we used an RT-PCR assay that we had previously used to measure the levels of Brn-3a/Brn-3b and other POU family transcription factors in limited amounts of material $(24,25)$. The levels of amplification product obtained after amplification of the Brn-3a and Brn-3b mRNAs with specific primers was compared to that of the constitutively expressed cyclophilin mRNA in each sample to control for any differences in the amount of total RNA or amplification efficiency in each sample (Fig. 1).

After densitometric scanning of results of this kind, the level of the Brn-3a and Brn-3b mRNAs was quantitated in 16 cervical samples derived from individuals with no histologically detectable cervical abnormality, and 14 CIN3 (CINIII) samples. The levels of mRNA observed in replicate determinations using the same sample varied by $<10 \%$, confirming the reliability of the assay (data not shown). The results of the analysis (Fig. 2 and Table I) revealed virtually no change in expression of Brn-3b mRNA between the normal and CIN3 samples with a similar mean level and an overlapping range of values between the two samples. In contrast, the mean level of the Brn-3a mRNA was elevated approximately 300-fold in the CIN3 samples when compared with the normals (Fig. 3 and

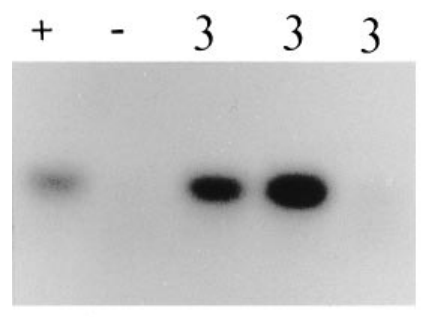

A
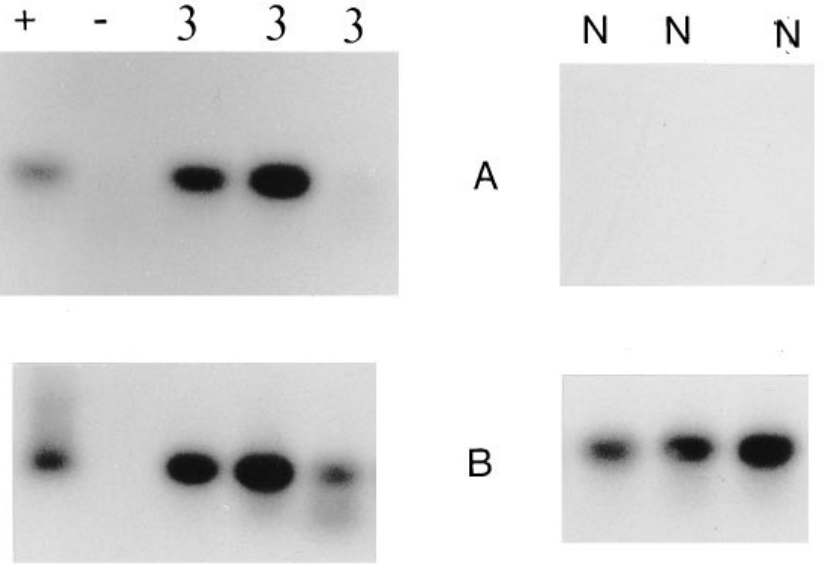

\section{B}
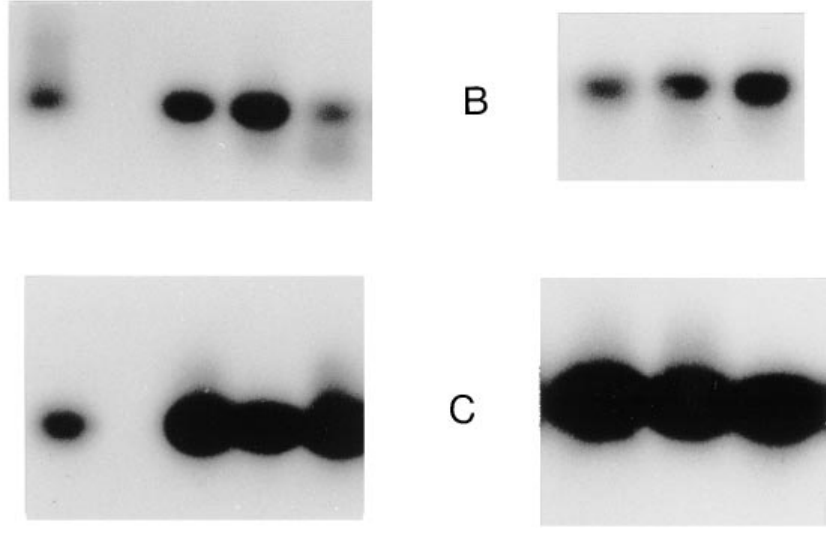

Figure 1. Results of RT-PCR amplification of the Brn-3a $(A), \operatorname{Brn}-3 \mathrm{~b}$ $(B)$, or control cyclophilin $(C)$ mRNAs in three samples obtained from women with no histologically detectable cervical abnormality $(N)$, or three samples from CIN3 lesions (3). The positive control of cDNA prepared from human neuroblastoma mRNA $(+)$ and the negative control of no cDNA (-) are also indicated. 


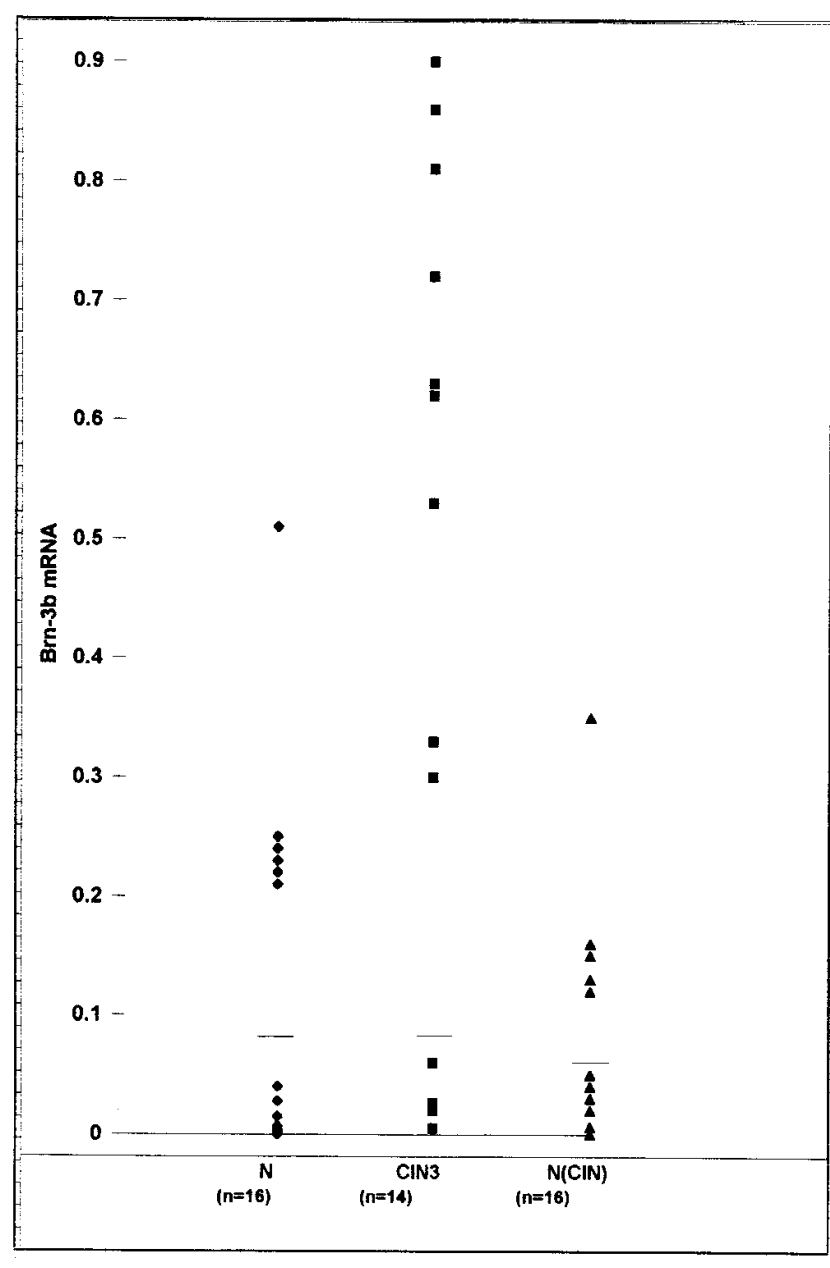

Figure 2. Brn-3b mRNA levels in cervical samples from women with no histologically detectable abnormality $(N)$, from CIN3 regions (CIN3), or from normal samples adjacent to a CIN3 lesion $(N-C I N)$. The horizontal bar shows the mean in each group, and the number of samples in each group is indicated $(n)$.

Table I). Indeed, the CIN3 samples with the lowest level of Brn-3a mRNA still had approximately 6-fold more mRNA than did the highest normal samples. The levels of Brn-3a mRNA were at the limit of detection in five of the normal samples, and were undetectable in the remainder.

These dramatic changes in the level of Brn-3a mRNA in the CIN3 samples compared with similar levels of Brn-3b resulted in a very large elevation in the Brn-3a:Brn-3b ratio in the CIN3 when compared with normal samples (Table I). As

Table I. Levels of Brn-3a and Brn-3b in Individuals With No Detectable Cervical Abnormality, CIN3 Lesions, and Regions Adjacent to a CIN3 Lesion ( $N$-CIN)

\begin{tabular}{lccc}
\hline & Normal & CIN3 & N-CIN \\
\hline Brn-3a & $0.001 \pm 0.003$ & $0.46 \pm 0.30$ & $0.42 \pm 0.31$ \\
Brn-3b & $0.1 \pm 0.11$ & $0.11 \pm 0.18$ & $0.07 \pm 0.09$ \\
Brn-3a/Brn-3b & 0.01 & 4.2 & 6.0 \\
Sample size & 16 & 14 & 16 \\
\hline
\end{tabular}

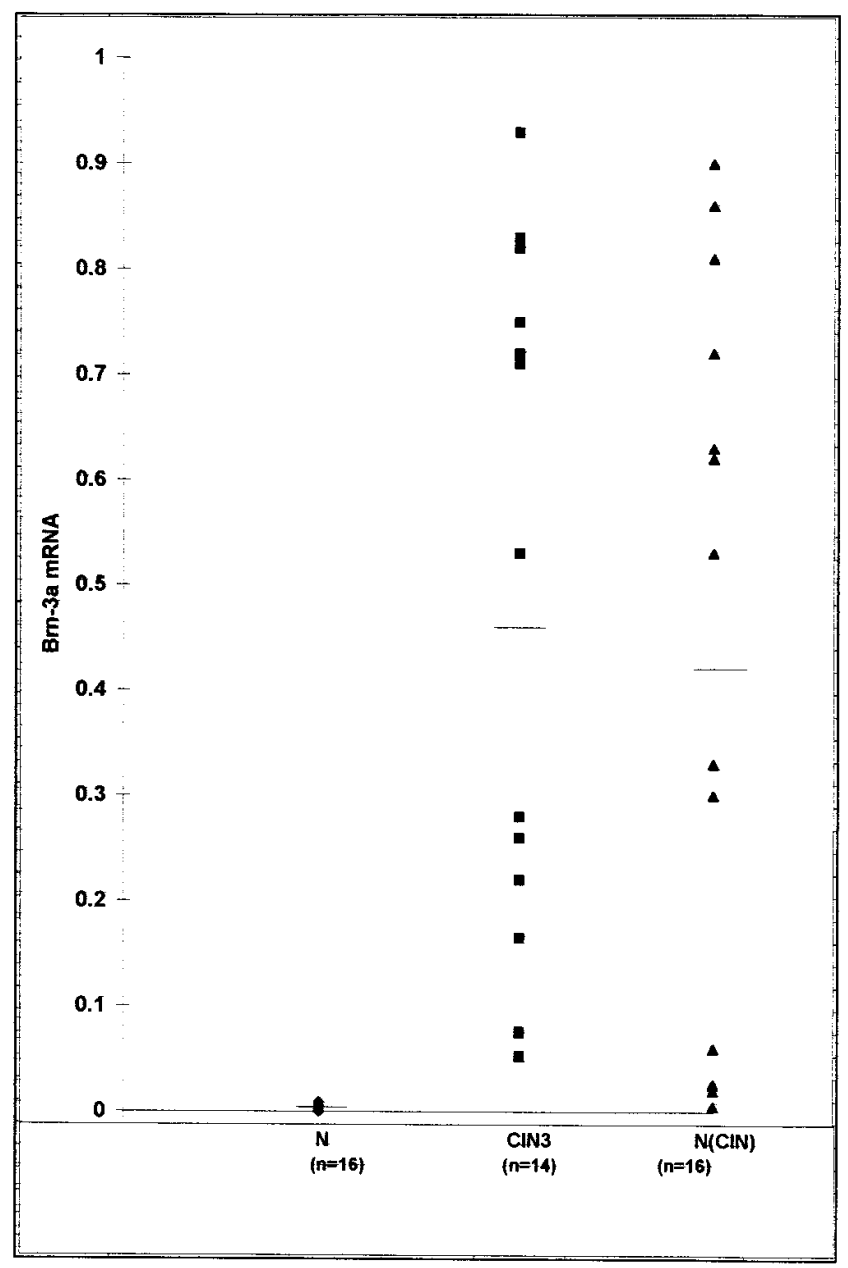

Figure 3. Brn-3a mRNA levels in the same samples as in Fig. 2.

the ratio between the Brn-3a activator and the Brn-3b repressor critically determines the activity of the HPV URR (19), it is likely that this effect plays a key role in activation of HPV gene expression in the CIN3 patients.

Evidently, this elevation of Brn-3a could either be confined to the CIN3 region of these patients, or could represent a more widespread elevation in the patients with CIN3 that also occurred in adjacent histologically normal regions of the cervix. To distinguish these possibilities, we determined the levels of Brn-3a and Brn-3b in further material from CIN3 patients that was derived from adjacent regions of the cervix with no detect-

Table II. Levels of Brn-3a mRNA HPV DNA and HPV RNA in a Single Individual With CIN3

\begin{tabular}{lccc}
\hline Section DNA level & Brn-3a mRNA level & HPV E6/E7 RNA level & HPV \\
\hline 1 & 0.075 & Undetectable & 0.87 \\
2 & 0.72 & 0.31 & 0.80 \\
3 & 0.30 & 0.04 & 0.90 \\
4 & 0.63 & 0.30 & 0.80 \\
\hline
\end{tabular}

Values are in arbitary densitometric units equalized for the level of cyclophilin mRNA in each sample. 


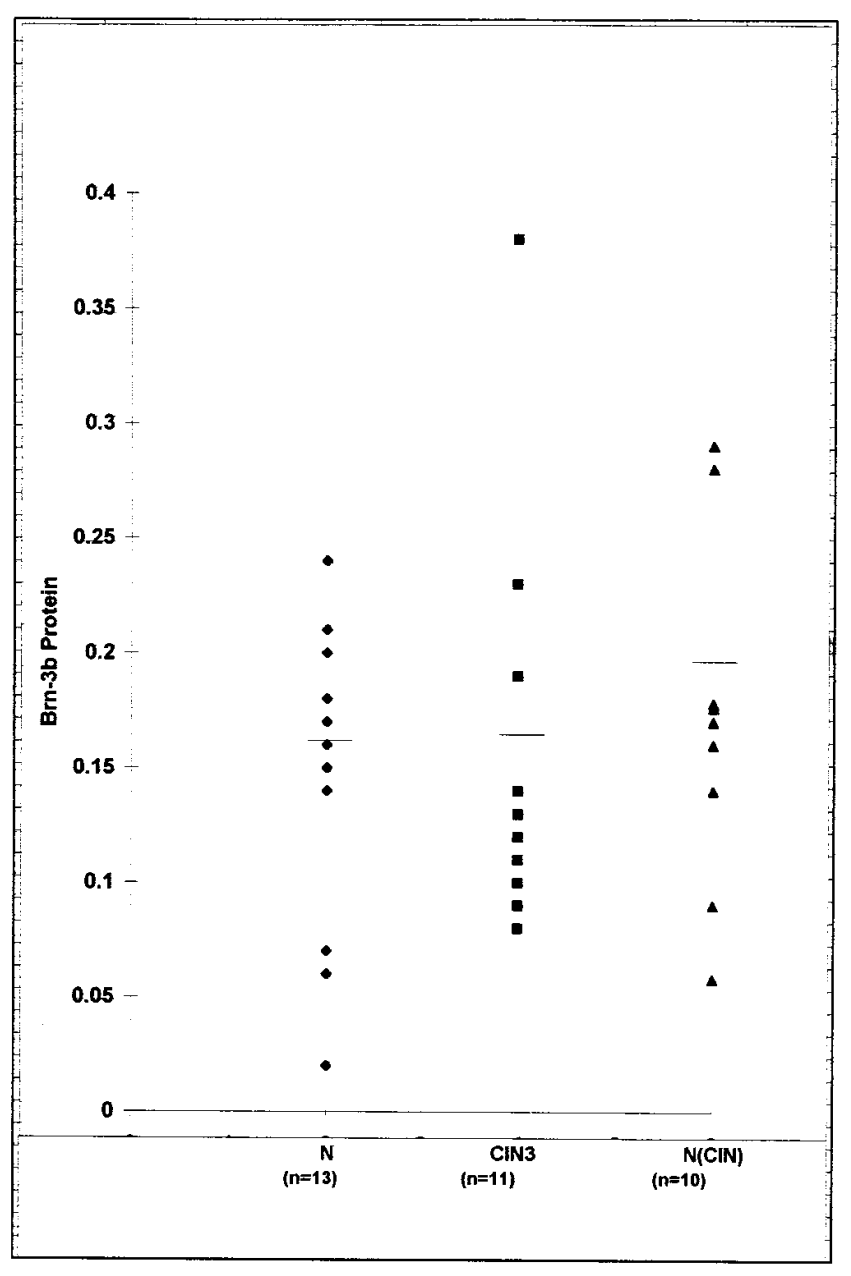

Figure 4. Brn-3b protein levels as determined by Western blotting of cervical samples from women with no histologically detectable abnormality $(N)$, from CIN3 samples (CIN3), or from normal samples adjacent to a CIN3 lesion $(N-C I N)$.

able abnormality, and that had no detectable expression of HPV E6 and E7 mRNA or HPV 16 DNA (data not shown). In these experiments, the samples exhibited a mean level of Brn-3a mRNA that was only marginally lower than that of the CIN3 region, and was dramatically more than what was detectable in the normal samples obtained from individuals with no histologically detectable abnormality anywhere in the cervix (Fig. 3 and Table I).

Together with a similar level of Brn-3b expression in these samples (Fig. 2), this resulted in a Brn-3a:Brn-3b ratio that was similar to that in the CIN3 samples, and was much greater than that of normal individuals (Table I). Hence, the elevated levels of Brn-3a and of the Brn-3a:Brn-3b ratio appear to be characteristic of both the CIN3 area and the adjacent area of the cervix of individuals with CIN3 compared with normal cervical tissue rather than of the actual area of malignancy alone.

To further extend these results to the protein level, we carried out Western blotting experiments using extracts from the various samples and antibodies to Brn-3a and Brn-3b. These results (Figs. 4 and 5) paralleled the results obtained at the mRNA level. Thus, the mean level of Brn-3b was similar in all the groups (Fig. 4), while the level of Brn-3a was greatly elevated in CIN3 samples and normal samples from adjacent regions compared with the results obtained with normal samples from women with no detectable cervical abnormality (Fig. 5).

Although virtually all the samples from CIN3 patients had a uniformly high level of Brn-3a, we did identify a patient in whom four adjacent CIN3 sections had different levels of Brn$3 \mathrm{a}$, including one having the lowest level detected in a CIN3 sample (Table II). This sample provided us with a unique opportunity to test the hypothesis that Brn-3a levels regulate transcription of the HPV genome. To do this, the level of HPV DNA and of HPV E6 and E7 RNA transcripts was measured using PCR amplification. In this experiment (Table II) all four regions had a similar level of HPV DNA, but the level of HPV E6 and E7 RNA transcripts detected was different in each case when compared with the level of the invariant cyclophilin mRNA. No signal was obtained with the HPV primers and DNA or mRNA prepared from an HPV-negative human neuroblastoma cell line, confirming the specificity of the assay. Most interestingly, HPV E6 and E7 RNA was undetectable in the sample with minimal Brn-3a levels, was present at low levels in the sample with intermediate Brn-3a levels, and was found at high levels in the two samples with high Brn-3a levels

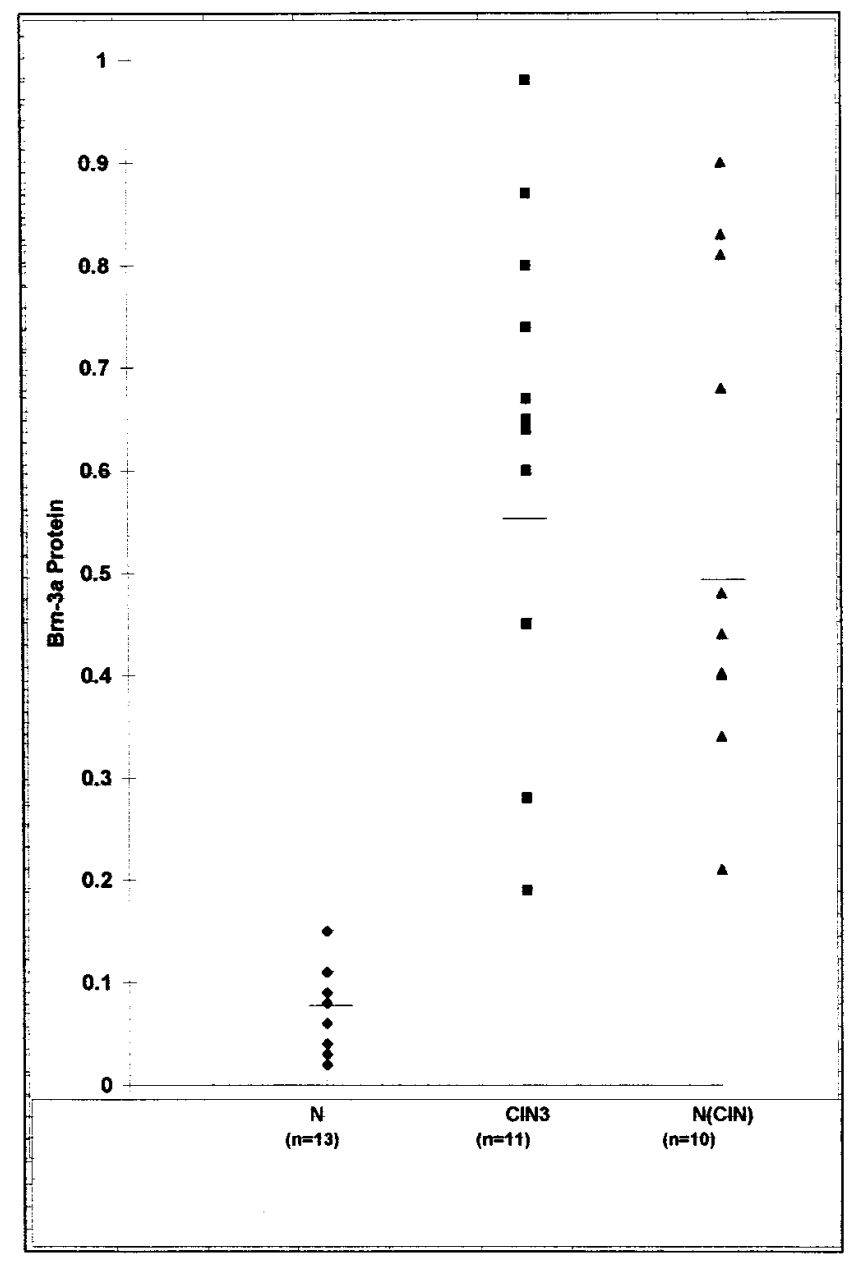

Figure 5. Brn-3a protein levels in the same samples as in Fig. 4. 
(Table II). As expected, the level of Brn-3b was similar in all the samples (data not shown).

\section{Discussion}

The data presented here indicate that the level of the Brn-3a cellular transcription factor increases in patients with CIN3 lesions compared with the levels observed in normal cervical cells from individuals with no detectable cervical abnormality. The very dramatic increase in the levels of Brn-3a contrast with the similar levels of Brn-3b in all the samples, and results in a rise in the Brn-3a:Brn-3b ratio. Since Brn-3a can activate via the HPV URR, whereas Brn-3b inhibits both its basal activity and its activation by Brn-3a (19), changes in this ratio are likely to play a critical role in regulating URR activity.

The elevated level of Brn-3a in the CIN3 samples compared to that in normal cells suggests a role for this elevation in the activation of HPV gene expression, and in the resulting cellular changes. On the basis of this initial comparison, however, it remained possible that the elevated level of Brn-3a was simply a consequence of the altered nature of these cells. Thus, in this case the elevation would reflect some property of the cells resulting from their transformation by HPV rather than being involved in the actual activation of HPV gene expression.

However, elevated expression of Brn-3a in histologicaly normal segments of the cervix adjacent to the CIN3 region was also observed compared with the level observed in normal samples from individuals with no detectable cercival abnormality. These samples did not exhibit any detectable HPV DNA or RNA in accordance with previous results (21). Hence, elevation of Brn-3a appears not to be characteristic solely of the premalignant cells in these individuals. This result may indicate that the region adjacent to the CIN3 lesion, although histologicaly normal, is also abnormal in that it overexpresses Brn-3a. Alternatively, our findings may reflect widespread elevation of Brn-3a in the cervix of individuals with CIN3, either due to exposure to a specific stimulus, or to differences within the human population in cervical Brn-3a levels.

In either case, it is clear that a dramatic elevation of Brn-3a levels occurs in the cervical cells of some individuals either throughout the cervix or in a relatively restricted region. In those individuals of this type also infected with HPV-16 and HPV-18, such an elevation will result in activation of the URR leading to E6 and E7 expression and cellular alterations in the cervical transformation zone at the junction of the endocervix and ectocervix where cervical tumors appear. In agreement with this idea, the level of HPV transcripts in different CIN3 samples from a single individual directly correlated with the level of Brn-3a in each sample. Hence, elevation of Brn-3a levels could play a critical role in activation of viral transcription and disease progression, although other factors such as viral type, viral load, and differences in cellular susceptibility to transformation would be responsible for precise localization of the malignant lesions.

These considerations focus attention on the stimuli that regulate Brn-3a expression in cervical cells. Although stimuli that can regulate the levels of Brn-3a and Brn-3b in opposite directions in neuronal cells have previously been defined (13, $23,28)$, such studies now need to be carried out in cervical cells. Alternatively, it is possible that the elevated Brn-3a lev- els in individuals with CIN3 reflect variation in the human population produced during cervical development and differentiation that affects HPV transcription. Whatever the case, it is clear that a dramatic elevation in Brn-3a levels is characteristic of women with high-grade cervical lesions compared with individuals with no detectable cervical abnormality.

\section{Acknowledgments}

This work was supported by Wellbeing (The Health Research Charity for Women and Babies).

\section{References}

1. Schiffman, M.H., H.M. Bauer, R.N. Hoover, A.G. Glass, D.M. Cadell, B.B. Rush, D.R. Scott, M.E. Sherman, R.J. Kurman, S. Wacholder, et al. 1983. Epidemiological evidence showing that human papillomavirus infection causes most cervical intraepithelial neoplasia. J. Natl. Cancer Inst. 85:958-964.

2. Durst, M., L. Gissmann, H. Ikenberg, and H.A. Zur Hausen. 1983. Papilloma virus DNA from a cervical carcinoma and its prevalence in cancer biopsy samples from different geographic regions. Proc. Natl. Acad. Sci. USA. 80: 3812-3815.

3. Cox, M.F., C.A. Meanwell, N.J. Maitland, G. Blackledge, C. Scully, and J.A. Jordan. 1986. Human papillomavirus type-16 homologous DNA in normal human ectocervix. Lancet. 2:157-158.

4. Meanwell, C.A. 1987. HPV16 DNA in normal and malignant cervical epithelium: implications for the etiology and behavior of cervical neoplasia. Lancet. 1:703-707.

5. Cripe, T.C., T.H. Hangen, J.P. Turk, F. Tabatabai, P.G. Schmid, M. Durst, L. Gissmann, A. Roman, and L. Twek. 1987. Transcriptional regulation of the human papilloma virus-16 E6-E7 promoter by a keratinocyte-dependent enhancer and by viral E2 transactivator and repressor gene products: implications for cervical carivogenesis. EMBO (Eur. Mol. Biol. Organ.) J. 6:3745-3753.

6. Gloss, B., H.U. Bernard, K. Seedorf, and G. Klock. 1987. The upstream regulatory region of the human papilloma virus contains an E2 protein-independent enhancer which is specific for cervical carcinoma cells and regulated by glucocorticoid hormones. EMBO (Eur. Mol. Biol. Organ.) J. 6:3735-3743.

7. Chan, W.-K., T. Chong, U. Benard, and G. Klock. 1990. Transcription of the transforming genes of the oncogenic human papilloma virus-16 is stimulated by tumour promoters through AP1 binding sites. Nucleic Acids Res. 18: 763-769.

8. Chan, W.-K., G. Klock, and H.-U. Bernard. 1989. Progesterone and glucocorticoid response elements occur in the long control regions of several human papilloma viruses involved in anogenital neoplasia. J. Virol. 63:3261-3269.

9. Gloss, B., M. Yeo-Gloss, M. Meisterenst, L. Rogge, E.L. Wimacker, and H.-U. Bernard. 1989. Clusters of nuclear factor 1 binding sites identify enhancers of several papillomaviruses but alone are not sufficient for enhancer function. Nucleic Acids Res. 17:3519-3533.

10. Nakshatri, H., M.M. Pater, and A. Pater. 1990. Ubiquitous and cell type specific protein interactions with human papillomavirus type 16 and type 18 enhancers. Virology. 178:92-103.

11. Dent, C.L., G.A.J. McIndoe, and D.S. Latchman. 1991. The constitutively expressed octamer binding protein OTF-1 and a novel octamer binding protein expressed specifically in cervical cells bind to an octamer-related sequence in the human papillomavirus 16 enhancer. Nucleic Acids Res. 19:45314535 .

12. Morris, P.J., C.L. Dent, C.J.A. Ring, and D.S. Latchman. 1993. The octamer binding site in the HPV16 regulatory region produces opposite effects on gene expression in cervical and non-cervical cells. Nucleic Acids Res. 21:10191023.

13. Gerrero, M.R., R.J. McEvilly, E. Turner, C.R. Lin, S. O'Connell, K.J. Jenne, M.V. Hobbs, and M.G. Rosenfeld. 1993. Brn-3.0 A POU domain protein expressed in the sensory, immune and endocrine systems that functions on elements distinct from known octamer motifs. Proc. Natl. Acad. Sci. USA. 90: 10841-10845.

14. Lillycrop, K.A., V.S. Budhram-Mahadeo, N.D. Lakin, G. Terrenghi, J.N. Wood, J.M. Polak, and D.S. Latchman. 1992. A novel POU family transcription factor is closely related to Brn-3 but has a distinct expression pattern in neuronal cells. Nucleic Acids Res. 20:5093-5096.

15. Turner, E.E., K.J. Jenne, and M.G. Rosenfeld. 1994. Brn-3.2: a Brn-3related transcription factor with distinctive central nervous system expression and regulation by retinoic acid. Neuron. 12:205-218.

16. Verrijzer, C.P., and P.C. van der Vliet. 1993. POU domain transcription factors. Biochim. Biophys. Acta. 1173:1-21.

17. Wegner, M., D.W. Drolet, and M.G. Rosenfeld. 1993. POU-domain proteins: structure and function of developmental regulators. Curr. Opin. Cell Biol. 5:488-498.

18. Theil, T., S. McLean-Hunter, M. Zornig, and T. Möröy. 1993. Mouse 
Brn-3 family of POU transcription factors: a new amino terminal domain is crucial for the oncogenic activity of Brn-3A. Nucleic Acids Res. 21:5921-5929.

19. Morris, P.J., T. Theil, C.J.A. Ring, K.A. Lillycrop, T. Möröy, and D.S. Latchman. 1994. The opposite and antagonistic effects of the closely related POU family transcription factors on the activity of a target promoter are dependent upon differences in the POU domain. Mol. Cell. Biol. 14:6907-6914.

20. Chirgwin, J.M., A.E. Przybyla, R.J. MacDonald, and W.J. Rutter. 1979. Isolation of biologically active ribonucleic acid from sources enriched in ribonuclease. Biochemistry. 18:5294-5299.

21. Ho, L., G. Terry, B. Mansell, B. Butler, and A. Singer. 1994. Detection of DNA and E7 transcripts of human papillomavirus types 16, 18, 31 and 33, TGF $\beta$, and GM-CSF transcripts in cervical cancers and precancers. Arch. Virol. 139:79-85.

22. Smith, M.D., S.J. Dawson, and D.S. Latchman. 1997. The Brn-3a transcription factor induces neuronal process outgrowth and the co-ordinate expression of genes encoding synaptic proteins. Mol. Cell. Biol. 17:345-354.

23. Budhram-Mahadeo, V.S., T. Theil, P.J. Morris, K.A. Lillycrop, T. Möröy, and D.S. Latchman. 1994. The DNA target site for the Brn-3 POU family transcription factors can confer responsiveness to cyclic AMP and removal of serum in neuronal cells. Nucl. Acids Res. 22:3092-3098.

24. Kendall, G., A. Brar-Rai, E. Ensor, J. Winter, J.N. Wood, and D.S Latchman. 1995. Nerve growth factor induces the Oct-2 transcription factor with the kinetics of an immediate-early gene. J. Neurosci. Res. 40:169-176.

25. Begbie, J.L., J.N. Wood, P.N. Anderson, and D.S. Latchman. 1996. Specific up-regulation of the POU domain transcription factor Oct-2 following axotomy. Neurosci. Lett. 207:185-186.

26. Bradford, M. 1976. A rapid and sensitive method for the quantitation of microgram quantities of proteins utilizing the principle of protein-dye binding. Anal. Biochem. 72:248-254.

27. Dhillon, V.B., S. McCallum, P.M. Norton, B.M. Twomey, F. ErkellerYuksel, P. Lydyard, D.A. Isenberg, and D.S. Latchman. 1993. Differential heat shock protein over-expression and its clinical relevance in systemic lupus erythematosus. Ann. Rheum. Dis. 52:436-442.

28. Budhram-Mahadeo, V.S., K.A. Lillycrop, and D.S. Latchman. 1995. The levels of the antagonistic transcription factors Brn-3a and Brn-3b in neuronal cells are regulated in opposite directions by serum growth factors. Neurosci. Lett. 185:48-51. 